\title{
OpenEnergySim: Conducting Behavioral Studies in Virtual Worlds for Sustainable Transportation
}

\author{
Helmut Prendinger, Arturo Nakasone, Mark Miska, and Masao Kuwarhara
}

\begin{abstract}
Intelligent Transport System (ITS) strategies can significantly reduce $\mathrm{CO}_{2}$ emissions of vehicles. Since the impact of ITS measures is highly dependent on driver acceptance and compliance rates, it is important to study the response of human drivers to new ITS strategies (the "human factor"). However, there is currently no low-cost yet effective method to investigate the human factor. Conventional driving simulators offer high realism of the driving and traffic environment, but they are expensive, not easily accessible, and restricted to one driver at a time, and hence make it difficult to conduct large-scale behavioral studies. Web-based survey methods are accessible but they do not allow to capture a person's moment-by-moment driving behavior. Therefore, we propose OpenEnergySim, an online multi-user three-dimensional (3D) simulation space based on emerging 3D Internet technology. OpenEnergySim can provide three functions in a realistic and integrated environment: (1) driving simulation of multiple users (as graphical 'avatars') in a simulated traffic network by using computer keyboard or game wheel; (2) visualization of the result of microscopic traffic simulation and (metaphoric) visualization of $\mathrm{CO}_{2}$ emissions; (3) a shared real-time collaboration space for testing and comparing the effects of "green" ITS strategies on $\mathrm{CO}_{2}$ emission reduction. Those features make OpenEnergySim a highly accessible platform for conducting behavioral studies in the transport domain. Since driving simulators have to address behavioral validity concerns, we will present results of a study where "car following" behavior in the virtual world is compared to both real-world data and Gipps' car-following model.
\end{abstract}

\section{INTRODUCTION}

In Japan, about $20 \%$ of $\mathrm{CO}_{2}$ emissions can be attributed to traffic. ${ }^{1}$ Intelligent Transport System (ITS) measures such as eco-driving, eco-routing, and information provision have the potential to induce energy-efficient driving and transportation, and consequently reduce $\mathrm{CO}_{2}$ emissions. However, the success of "green" ITS measures crucially depends on drivers' compliance and acceptance rates (the "human factor").

Therefore, effective tools for estimating the effect of "green" ITS strategies on $\mathrm{CO}_{2}$ emission are required. Conventional methods such as high-tech driving simulators (e.g. [1], [2], [3], [4]) are inadequate because of their high cost and thus low accessibility, which makes behavioral data

H. Prendinger is with the National Institute of Informatics, 2-1-2 Hitotsubashi. Chiyoda-ku, Tokyo 101-8430, Japan helmuteni .ac.jp

A. Nakasone is with the National Institute of Informatics, 2-1-2 Hitotsubashi. Chiyoda-ku, Tokyo 101-8430, Japan arturonakasoneenii.ac.jp

M. Miska is with the Smart Transport Research Center, Queensland University of Technology, Gardens Point Campus, GPO Box 2434 Brisbane, QLD 4001, Australia marc.miska@qut.edu.au

M. Kuwahara is with Tohoku University, Aoba, Aoba-ku, Sendai, Miyagi 980-8579, Japan kuwahara@plan.civil.tohoku.ac.jp

${ }^{1}$ Japan for Sustainability (JFS) Newsletter No. 95, July 2010 collection difficult. On the other hand, web-based surveys (e.g. [5]) provide high accessibility, but restrict the type of data obtained to high-level decision making on route choice. This limitation leaves out instantaneous driving behavior such as acceleration or deceleration, which is important to assess the energy-efficiency of driving [6].

To overcome the limitations of conventional methods, we propose OpenEnergySim as a novel behavioral data collection platform for studying the effect of ITS strategies on driving behavior, with the goal of $\mathrm{CO}_{2}$ emission reduction [7]. OpenEnergySim is an ITS application based on emerging 3D Internet technology [8], in particular the open source OpenSim virtual world simulator. ${ }^{2}$ OpenSim is a networked (online) multi-user three-dimensional (3D) virtual world, where users are represented as 'avatars' (graphical selfrepresentations) that can interact with each other and objects in the virtual worlds, such as (virtual) cars or road furniture. OpenEnergySim has the following salient features.

- Multi-User Immersive Driving. Multiple users can connect to OpenEnergySim simultaneously and participate ('immerse') as drivers by means of their avatars.

- High Accessibility. OpenEnergySim can be accessed easily by Internet and unlike most game engines, there are no licensing issues.

- Realistic Visualization and Integration of Simulation Results. The result of the simulations of traffic, pedestrians, environmental conditions, etc can be visualized with a high level of realism. Traffic simulation is synchronized with users' driving behavior.

- Shared Collaborative Space. Users can collaborate on testing new ITS strategies based on shared visualizations of the traffic scenario.

The multi-user driving feature is important, since driving behavior is highly influenced by the behavior of surrounding vehicles. In OpenEnergySim, we can study the inter-driver behavior among user-controlled cars. Together with the accessibility feature, conducting large-scale behavioral studies becomes possible, which is important to obtain sound results on the impact of ITS. The visualization of multiple trafficrelated phenomena in the virtual world supports a 'holistic' view on the research problem. The integration of immersive user driving with simulated traffic allows us conduct realistic traffic scenarios involving a large number of vehicles [9]. In addition, multiple users can experience the effect of "green" ITS strategies in different roles, as drivers, pedestrians, researchers, and so on, with their different interests, such as

\footnotetext{
${ }^{2} \mathrm{http} / / /$ opensimulator.org/
} 
convenience, life quality (air pollution, noise, etc), or safety.

OpenEnergySim leverages our previously developed technologies for:

- virtual world scenario creation [10], [11],

- large-scale data visualization in virtual worlds [12], and

- user studies in virtual worlds [13], [14], [15].

The remainder of the paper is organized as follows. In Section II, related work on conducting behavioral studies with driving (and travel) simulators will be discussed. Section III will explain the key components and features of the OpenEnergySim application. In Section IV, the "car following" experiment will be described. Section V summarizes and concludes the paper.

\section{RELATED WORK}

To estimate the impact of new ITS measures such as eco-friendly driving and routing, or "Park \& Ride", it is important to analyze the response of drivers to those ITS measures. Several researchers investigate the "human factor" by conducting dedicated driver behavior studies. The main methods are: (1) driving simulator experiments, and (2) interactive web-based surveys.

Several car companies and research institutes in USA and Europe have developed driving simulators [1], [2], [16], [4]. In Japan, a driving simulator has been developed at the University of Tokyo [3] as part of the "Sustainable ITS Project" [17]. Driving simulators aim at high realism of the driving experience by providing hardware installations such as a cockpit of a real car and a sophisticated high-tech display system. The driving simulator of the University of Tokyo has been used to conduct behavioral studies on sag and merging/diverging sections on expressways in Japan [18].

Although driving simulators provide detailed information about the driver's moment-by-moment driving behavior and reaction to ITS measures, they have serious limitations: (1) the cost of constructing and maintaining a driving simulator is prohibitively high, and (2) only one person at a time can experience the simulation. As a consequence of the first limitation, it is difficult to obtain the sample size necessary to draw valid conclusions from the experiment. Since driving behavior is highly influenced by the behavior of surrounding vehicles, it is desirable to let multiple human drivers participate in the experiment at the same time. This is not possible with currently available driving simulators.

To overcome the problem of small sample size, researchers started to use interactive web-sites as an alternative to driving simulators. At the Delft University of Technology, a travel simulator was developed in the Travel Simulator Laboratory [5]. The travel simulator is embedded in an interactive website that provides choices about travel routes to the user. The user may experience the effect of route choice by imposed waiting times of the travel session. For instance, a congestion will slow down the progress of the travel session.

A web-based simulator is well suited to investigate drivers' high-level decision making about ITS installments, such as choosing a route. Because of the high accessibility of websites, it is easy to collect data from a large group of people.
However, this approach has the following restrictions: (1) it is not designed to capture a driver's instantaneous driving behavior and (2) the level of realism to mimic the actual driving experience is low.

An important component of behavioral studies in the transport domain are traffic simulators that can generate the movement of individual cars (microscopic traffic). There exist several commercial software packages for simulating and visualizing microscopic traffic, including the VISSIM (PTV) or AIMSUN (TSS) developed in Europe (see [6] for an overview), or Avenue (i-Transport Lab) developed in Japan [19]. While those systems are full-fledged environments for traffic engineering, the main purpose of their 3D visualization component is advertisement and presentation, rather than driving experiments. An exception is the commercial software UC-win/Road, which integrates traffic simulation with different types of driving simulators [20]. However, as a stand-alone application, driving is restricted to a single user (driver).

A different perspective on integrating traffic simulation and individual driving behavior has been taken by researchers at Kyoto University [21]. First, behavior models are obtained by (i) logging data from a driving simulator and (ii) interviews to distill driving rules. Then, the resulting driver behavior models are fed into a multi-agent based traffic simulation, and their effect on traffic patterns is analyzed. Researchers at IBM Research Tokyo have developed MASMITS ("Metropolitan-Area Scale Microscopic Traffic Simulator"), an agent-based traffic simulator which is claimed to simulate millions of cars [22]. Similar to the Kyoto group, the goal of the IBM researchers is to integrate different driver models with the traffic simulator and study its overall effect, e.g. on the road network of Kyoto. This approach is now also being applied to investigating the impact of driving behavior on $\mathrm{CO}_{2}$ emission at city level [23]. Note that the purpose of those researches is different from ours. In our approach, driving of multiple users and traffic is integrated in one consistent simulation space (to analyze the "human factor"), whereas those works obtain a driver model independently of the targeted traffic simulation, and combine driver model and traffic simulation in a second step.

As a method for sustainable transportation, we aim to analyze the impact of "green" ITS measures on human driving behavior, which will lead to more effective ITS measures. On the other hand, the group at IBM [23] investigates the effect of energy-reducing measures (e.g. traffic policies) on the traffic flow of a city-wide area.

\section{OPENENERGYSIM}

OpenEnergySim [24] is a software platform for simulation and testing in the field of sustainable transport, based on the OpenSim virtual world simulator and OpenLibraryGrid, ${ }^{3}$ an extensive set of technologies for content creation in virtual worlds, developed at the National Institute of Informatics.

\footnotetext{
${ }^{3}$ http://www.globallabproject.net
} 


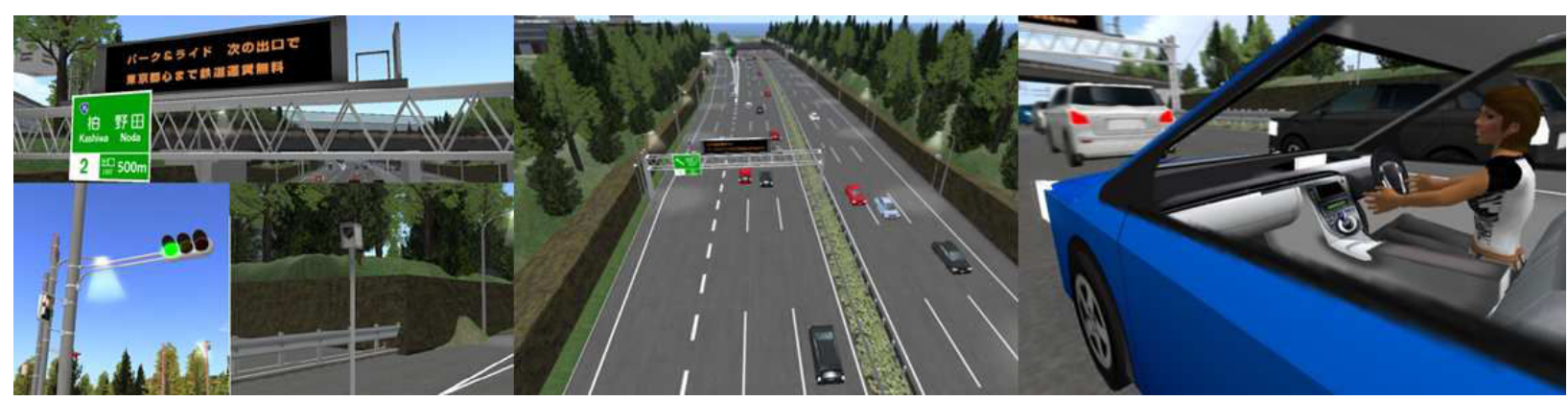

Fig. 1. Left: road furniture (traffic light, (variable) message sign, sensor). Middle: persistent traffic simulation. Right: immersive driving.

As a first application scenario, we modeled a part of Kashiwa, a prefecture of Tokyo, as a 3D virtual world (see Fig. 1). The purpose is to collect data on (1) driving behavior changes induced by online information on ecofriendly driving and (2) travel behavior changes due to the "Park \& Ride" ITS measure. The latter ITS strategy is expected to re-direct traffic from the highway (Interchange) to Lalaport, where drivers can park their car and take a train to Tokyo for free. OpenEnergySim is now in its testing phase as an environment for estimating the effect of ITS measures on $\mathrm{CO}_{2}$ emission reduction.

We will now describe the main modules and components of OpenEnergySim. Since OpenEnergySim is based on multi-user networked virtual worlds technology, its architecture consists of a server module and a client module. The responsibility of the server module is to coordinate the traffic simulator and its visualization in OpenSim, while accepting input from client-side user interaction, specifically users' driving behavior. The most important server components are:

- The X-Roads traffic simulator (TS) component generates the trajectories of individual vehicles (microscopic traffic) based on established traffic models [25], [9].

- The data rate converter component handles the data synchronization between the TS and user driven vehicles in the virtual world. Integrating server-side computer generated traffic and client-side user driving is a challenging task in a networked environment due to network lags between the server and the distributed clients. We use prioritization and merging techniques to achieve a consistent user (driving) experience for all clients participating in the simulation.

- The $\mathrm{CO}_{2}$ emission simulator generates (metaphoric) visualizations of emission. We currently employ 'cloud' animations above some key areas (highway, intersection, etc) that can change color and size depending on the emission level of the cars within the specified area.

- The user interface component supports the dynamic manipulation of road furniture (message signs, sensors, etc) and user interface elements such as a (interface) 'button' to create a user-controlled car.

- The pedestrian simulator generates movements of 'bots' (agents controlled by a computer program rather than the user). Currently, it is used to demonstrate the effect of increased traffic on pedestrians in Lalaport, the destination of "Park \& Ride" ITS measure.

- The event logging component stores the information about computer-controlled and user-controlled cars (position, velocity) and road furniture (variable message signs, traffic lights) in a central database. This information is used to analyze users' instantaneous driving behavior and route choice.

An attractive feature of OpenEnergySim is that traffic and user driving are generated at the same scale (resolution), which may immerse the user in the traffic situation. However, the level of immersion will greatly depend on the users' actual driving experience in the virtual world. This is the responsibility of the client module, which consists of two main components.

- The vehicle physics engine governs the vehicle's motion dynamics.

- The driving (user) interface handles the hardware (game wheel or keyboard) that users operate to control the virtual car.

The users' driving experience crucially depends on the smooth interplay between those two components. In computer game applications, both components are hosted in the client machine, which has the advantage of easy integration, but often suffers from licensing issues of the game software and hence, restricted accessibility. In a networked environment like OpenSim, a generic physics engine is located on the server, while the dynamic force values are calculated by the vehicle's client-side physics engine.

\section{CAR-Following ExPeriment}

The purpose of our experimental study was to determine the suitability of OpenEnergySim for collecting realistic behavioral data of drivers. To validate driving in OpenEnergySim, we chose 'car-following' as a simple yet common task for which both real-world data and mathematical models are available. Thus, we are interested in behavioral rather than physical validation of driving in OpenEnergySim. Behavioral validation refers to the degree that the simulator induces the same behavior of the driver as would be shown in the real world under similar circumstances [4]. ${ }^{4}$

\footnotetext{
${ }^{4}$ Physical validity measures the level to which the simulator reproduces the dynamics of the vehicle being modeled.
} 


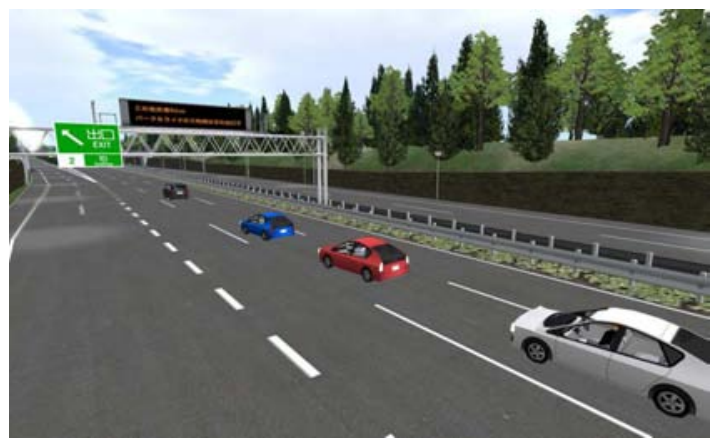

Fig. 2. Observer's view on the setup, where each of the three human drivers follows the car in front, forming a row of four cars. The lead car is computer-controlled and determines the route.

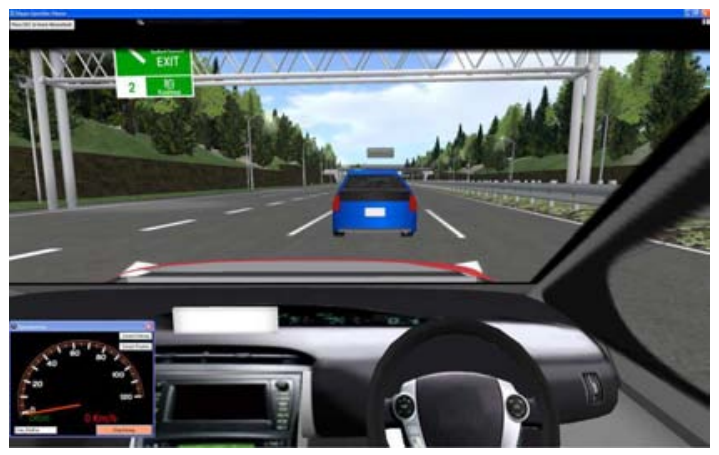

Fig. 3. Visualization of the experiment from the subject's perspective. On the bottom-left of the screen, a speedometer is located.

In line with [26], we understand validity as a taskdependent concept. Hence, our hypothesis is that we can reproduce realistic car-following behavior in the virtual world. This is important since the generated data can be used to calibrate simulation models for new technologies such as driver assistance systems, or situations that are hard to observe (rubber necking, sag curves, etc).

The generated data will be compared to real-world data and Gipps' model [27]. Regarding the first type, the behavior of the (computer-controlled) lead car has been extracted from trajectory data of the Interstate 80 (I-80) freeway data-set, provided by the Federal Highway Administration (Publication number: FHAW-HRT-06-137).

\section{A. Design and Procedure}

As a testing environment, we used a part of virtual Kashiwa (see Sect. III), which consists of two highway sections joined together by close curves at the extremes. As traffic engineers considered a study with three drivers ('followers') as an optimal number for analyzing car-following behavior, three subjects were invited to the study. Their task was to follow the car in front at a safe distance (whereby the first user-controlled car followed the computer-controlled lead car). In order to obtain sufficient data for behavioral comparison, the drivers were asked to drive for 3 hours and 15 minutes. Figure 2 depicts the experiment from an observer's perspective and Fig. 3 shows the view the secondlast driver at the same moment.

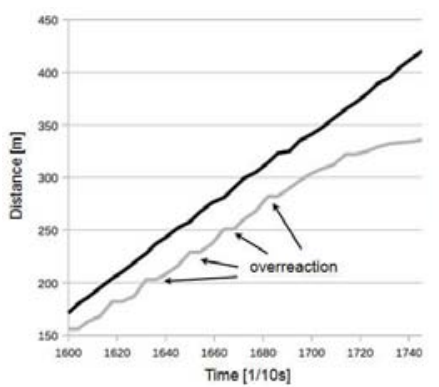

(a)

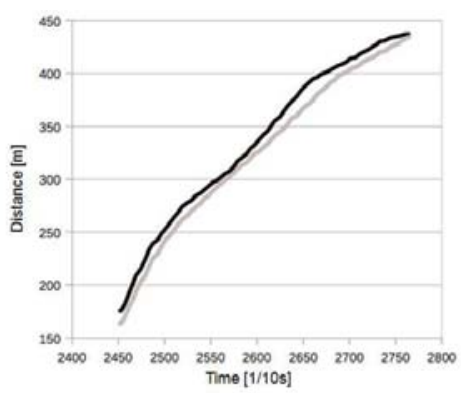

(b)
Fig. 4. Car following with constant speed at different following distances At larger distance, the following vehicle overreacts when decelerating (a), whereas at very close distance the following vehicle travels smoothly (b).

Subjects were seated in front of 23 inch screens and drove the virtual cars using Logitech G27 Gaming Wheels. ${ }^{5}$ To drive a car, the subjects simply had to instantiate ("fetch") a car by pressing a dedicated button in the environment, and then press the "Drive" option. This operation seats the user's avatar in the virtual car. Driving is then activated by setting the gear and acceleration with the gas pedal.

\section{B. Results}

We logged the $x, y$, and $z$ positions of the user-driven vehicles as well as the status of the steering wheel and driving pedals to a database. Because the raw position data are in three-dimensional space, data had to be transformed into a time-space relationship. This was achieved by transforming the coordinates into the distance traveled on the lane of the link. Using an algorithm for map matching of global positioning system (GPS) information, all trajectories were transformed into time-space relationships.

Then we used a time window to select the vehicles in the database that are following directly behind each other. In a last step, data from the end of the highway tracks were removed to avoid corruption by synthetic conditions (the curves). Effectively, we obtained about 100,000 data points, which translate into about one hour recorded driving for each of the three cars.

1) Comparison to Real-World Data: We have examined car-following behavior from the recorded data with respect to one-to-one following, braking maneuvers, and more complex interaction between the vehicles.

The data show that short following distances result in very smooth following behavior, while larger following distances seem to cause the following vehicle to overreact on headway changes. The overreaction decreases with more practice in the driving environment, but can still be clearly seen in Fig. 4(a), whereas such disturbances are absent in Fig. 4(b).

To investigate the roots of this phenomenon, we have checked trajectory data from braking scenarios. The first scenario is an emergency braking caused by a breakdown on the I-80, which was also experienced by the subjects in the experiment.

\footnotetext{
${ }^{5} \mathrm{http}: / /$ www.logitech.com
} 


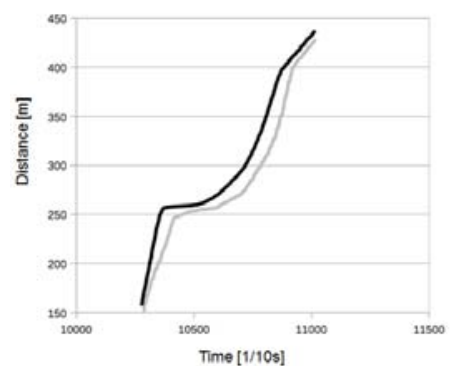

(a)

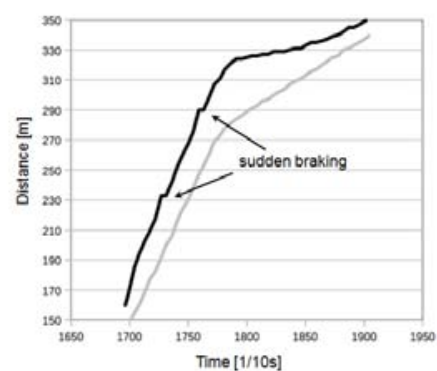

(b)
Fig. 5. Trajectories of braking scenarios. During an emergency brake of the leading vehicle, the following vehicle reacts quickly enough and can recover to normal following behavior after a short time (a). Sudden short brakes do not cause any overreaction of the follower, but lead to an increased following distance (b).

Figure 5(a) shows the trajectories of two following vehicles. The vehicles can avoid an accident and show normal recovery of speed after the incident. During the recovery phase the headway of the cars is increased, but recovers completely when constant traveling speed is restored. Thus, the braking behavior appears normal and leads to expected trajectories. Hence we speculate that the overreaction has its roots in the perception of distance in the simulator.

Therefore we looked into the effect of the above mentioned overreaction on following vehicles. By isolating a vehicle that shows sudden braking due to overreaction on headway changes, we found that cars following such a vehicle do not experience a disruption of smooth flow, but that the following-distance increases significantly in the period of the sudden braking with a substantial recovery time (see Fig. 5(b)). This indicates that the following-behavior of single-lane traffic is reproduced well in the experiment. The overreaction phenomenon needs to be addressed in more detail in future work.

As a traffic situation for a more complex interaction, we have simulated a highway on-ramp. Two vehicles are traveling on the highway and a third vehicle will merge into traffic from the on-ramp. Before merging, the entering vehicle will pass the second vehicle on the highway on the acceleration lane. This test showed that the merging action has an effect on the highway traffic. While the first vehicle keeps cruising at normal speed, the following vehicle decreases its speed when becoming aware of the merging vehicle. This phenomenon is well known and a partial cause of capacity drops at on-ramps. Figure 6 shows the trajectories of all three vehicles during the merging process. After the merging was completed, the three vehicles on the highway maintain a safe following distance in smooth following behavior. The result of this more complex situation is that interactions between cars on different lanes can be captured, and thus, multi-lane experiments can be conducted in the virtual environment.

Having the data checked by face value, we will now turn to checking the data against an existing model for driving behavior.

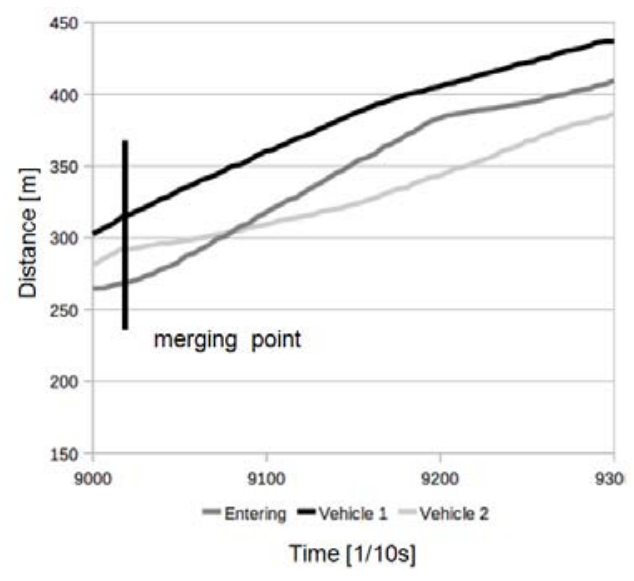

Fig. 6. Trajectories at a merging point where a vehicle enters a highway after passing a vehicle on the acceleration lane. While the leading vehicle cruises along the highway, the two following vehicles negotiate a following distance.

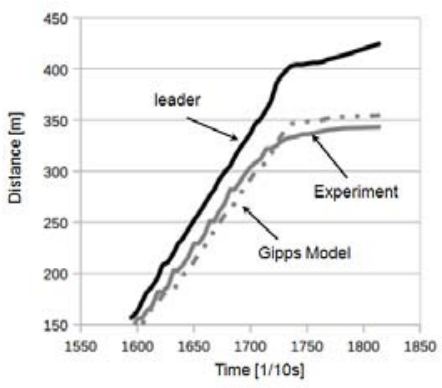

(a)

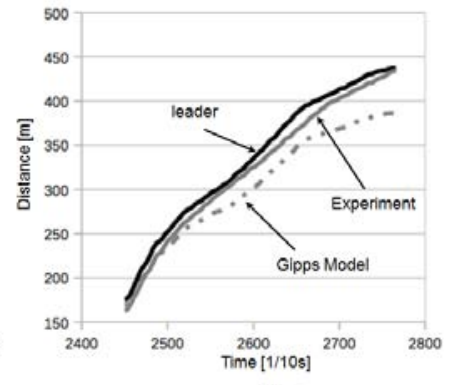

(b)
Fig. 7. Comparison of the experiment outcome with the following behavior described by the Gipps car following model. If drivers keep a safe distance the following behavior is similar, but the experiment can produce unrealistic short following distances due to the lack of feeling danger.

2) Comparison to Gipps' Car-Following Model: With the given data, a first-order car-following model like the one from Gipps [27, p. 107] is the most appropriate candidate for comparison. Applying the model to the data used before, one can see that the Gipps model reacts similar to the recorded data. This is shown in Fig. 7(a). The main difference is that the drivers in the experiment sometimes chose smaller following distances (see Fig. 7(b)). This finding can be explained by two reasons: (1) drivers in the simulator have no feeling of danger, and (2) the Gipps model-a collision avoidance model—specifically avoids dangerous situations.

\section{CONCLUSION}

We have presented OpenEnergySim, a platform for conducting behavioral driver studies based on emerging 3D Internet technology. In OpenEnergySim, multiple drivers can participate simultaneously by simply connecting to the OpenSim virtual world simulator. The purpose of our experimental space is test the impact of ITS measures on the reduction of $\mathrm{CO}_{2}$ emission. A part of a prefecture of Tokyo has been set up as a testing environment, and we will conduct a largescale study in the near future.

In order to estimate behavioral validity of our environment, we have conducted a preliminary study on "car following". 
The results of this experiment demonstrate the effectiveness of virtual worlds for driving experiments. Critical issues are the car-following at (dangerously) close distances (Fig. 4(b)) and the overreaction in terms of deceleration (Fig. 4(a)). Although we could show that the effects are relatively small (Fig. 5), these deviations would matter if used to calibrate car-following models. Another issue is lateral instability of driving in the virtual world due to a certain insensitivity of steering. This problem has to be solved by optimizing the communication between the game wheel operation and its realization in driving in-world. We will address these issues in our future work.

In the course of our studies on sustainable transportation, we will also integrate OpenEnergySim with major $\mathrm{CO}_{2}$ emission models, such as the Comprehensive Modal Emission Model (CMEM) ${ }^{6}$ developed at the UC Riverside, and the Avenue traffic simulator ${ }^{7}$ developed at i-Transport Lab in Japan.

\section{ACKNOWLEDGMENT}

The authors would like to thank Juan Camilo Ibarra, Martin Lindner, Kugamoorthy Gajananan, and Marconi Madruga from NII for their invaluable contribution to the implementation of OpenEnergySim and to the experiment.

\section{REFERENCES}

[1] J. Kuhl, D. Evans, Y. Papelis, R. Romano, and G. Watson, "The Iowa driving simulator: An immersive research environment," Computer, vol. 28, no. 7, pp. 35-41, 1995.

[2] S. Bayarri, M. Fernandez, and M. Perez, "Virtual reality for driving simulation," Communications of the ACM, vol. 39, no. 5, pp. 72-76, 1996.

[3] Y. Suda, Y. Takahashi, M. Kuwahara, S. Tanaka, K. Ikeuchi, M. Kagesawa, T. Shraishi, M. Onuki, K. Honda, and M. Kano, "Development of universal driving simulator with interactive traffic environment," in Proceedings of Intelligent Vehicles Symposium. IEEE, 2005, pp. 743-746.

[4] A. Hoskins and M. El-Gindy, "Technical report: Litature survey on driving simulator validation studies," International Journal of Heavy Vehicle Systems, vol. 13, no. 3, pp. 241-252, 2006.

[5] C. G. Chorus, E. J. Molin, T. A. Arentze, S. P. Hoogendoorn, H. J. Timmermans, and B. V. Wee, "Validation of a multimodal travel simulator with travel information provision," Transportation Research Part C, vol. 15, pp. 191-207, 2007.

[6] A. Spence, S. Turksma, A. Schelling, T. Benz, J.-P. Medevielle, and I. McCrae, "Methodologies for assessing the impact of ITS applications on $\mathrm{CO}_{2}$ emissions," EC-METI Task Force, Technical Report V1.0, March 2009.

[7] M. Miska, H. Prendinger, A. Nakasone, and M. Kuwahara, "Driving and traveller behavior studies using 3D Internet," in Proceedings 13th International IEEE Conference on Intelligent Transportation Systems (ITSC'10). IEEE, 2010.

[8] W. Bainbridge, "The scientific research potential of virtual worlds," Science, vol. 317, pp. 472-476, 2007.

[9] T. Jiang, M. Miska, M. Kuwahara, A. Nakasone, and H. Prendinger, "Microscopic simulation for virtual worlds with self-driving avatars," in Proceedings 13th International IEEE Conference on Intelligent Transportation Systems (ITSC'10). IEEE, 2010.

[10] A. Nakasone and H. Prendinger, "EML3D: An XML based markup language for 3D object manipulation in Second Life," in Proceedings 9th International Symposium on Smart Graphics (SG'09). Springer LNCS 5531, 2009, pp. 263-272.

\footnotetext{
${ }^{6}$ http://www.cert.ucr.edu/cmem/

${ }^{7}$ http://www.i-transportlab.jp/
}

[11] H. Prendinger, S. Ullrich, A. Nakasone, and M. Ishizuka, "MPML3D Scripting agents for the 3D Internet," IEEE Transactions on Visualization and Computer Graphics, 2010, in press.

[12] A. Nakasone, H. Prendinger, S. Holland, P. Hut, J. Makino, and K. Miura, "Astrosim: Collaborative visualization of an astrophysics simulation in Second Life," IEEE Computer Graphics and Applications, vol. 29, no. 5, pp. 69-81, 2009.

[13] H. Prendinger, "The Global Lab: Towards a virtual mobility platform for an eco-friendly society," Transactions of the Virtual Reality Society of Japan, vol. 14, no. 2, pp. 163-170, 2009.

[14] H. Prendinger, B. Brandherm, and S. Ullrich, "A simulation framework for sensor-based systems in Second Life," Presence: Teleoperators and Virtual Environments, vol. 18, no. 6, pp. 468-477, 2009.

[15] S. Attasiriluk, A. Nakasone, W. Hantanong, R. Prada, P. Kanongchaiyos, and H. Prendinger, "Co-presence, collaboration, and control in environmental studies. a Second Life based approach," Virtual Reality, vol. 13, no. 3, pp. 195-204, 2009.

[16] E. Blana, "Driving simulator validation studies: A literature review," Institute of Transport Studies, University of Leeds, Tech. Rep. Working Paper 480, 1996.

[17] K. Ikeuchi, M. Kuwahara, Y. Suda, Y. Tanaka, E. Chung, T. Suzuki, M. Kagesawa, S. Tanaka, I. Nishikawal, Y. Takahashi, R. Horiguchi, T. Shiraishi, H. Hanabusal, H. Kawasaki, H. Ishikawa, K. Maruoka, K. Honda, M. Furukawa, M. Kanos, H. Ueno, Y. Ohba, Y. Mashiyama, T. Oda, K. Kenmotsu, T. Yamamoto, M. O. Masaaki, M. Sakai, and M. Tsuji, "Sustainable ITS project overview: Mixed reality traffic experiment space under interactive traffic environment for ITS," in Proceedings of Intelligent Vehicles Symposium. IEEE, 2005, pp. 735738.

[18] K. Honda, T. Shiraishi, M. Onuki, M. Kano, M. Kuwahara, K. Ikeuchi, Y. Suda, and M. Kasegawa, "Evaluation of driving behavior using virtual reality experiment at expressway sag and merging/diverging sections," in Proceedings of ITS World Congress 2005, 2005, paper 3034.

[19] R. Horiguchi, M. Kuwahara, M. Katakura, H. Akahane, and H. Ozaki, "A network simulation model for impact studies of traffic management avenue ver.2," in Proceedings 3rd Annual World Congress on ITS, 1996.

[20] T. Lorentzen, Y. Kobayashi, and Y. Ito, "Virtual reality driving simulation: Integrating infrastructure plans, traffic models, and driving behaviors," in 2009 Annual Meeting and Exposition of the Intelligent Transportation Society of America, 2009.

[21] Y. Nakajima, Y. Nakai, H. Hiromitsu, and T. Ishida, "Wide-area traffic simulation based on driving behavior model," in Proceedings of PRIMA 2009. Spinger LNAI 5925, 2009, pp. 459-470.

[22] S. Kato, G. Yamamoto, H. Mizuta, and H. Tai, "Simulating whole city traffic with millions of multiple driver cars," IBM RT0759, Tech. Rep., 2009.

[23] S. Kato, "PREDICT project: Research and developement of a largescale mobility society simulator for the reduction of vehicle $\mathrm{CO}_{2}$ emission," 2010, presentation slides, IBM Research Tokyo.

[24] A. Nakasone, H. Prendinger, M. Miska, M. Lindner, R. Horiguchi, J. C. Ibarra, K. Gajananan, R. Mendes, M. Madruga, and M. Kuwahara, "Openenergysim: A 3D Internet based experimental framework for integrating traffic simulation and multi-user immersive driving," in Proceedings 4th International Conference on Simulation Tools and Techniques (SIMUTools'11), Industry Track, 2011.

[25] M. Miska, "Real time traffic management by microscopic online simulation," TRAIL Thesis Series, The Netherlands, Tech. Rep. T2007/1, 2007.

[26] N. A. Kaptein, J. Theeuwes, and R. van der Horst, "Driving simulator validity: Some considerations," Transportation Research Record, vol. 1550, pp. 30-36, 1996.

[27] P. Gipps, "A behavioural car-following model for computer simulation," Transportation Research Board Part B, vol. 15, pp. 105-111, 1981. 